\title{
LEVEL CROSSINGS OF A RANDOM POLYNOMIAL WITH HYPERBOLIC ELEMENTS
}

\author{
Submitted for publication to the \\ Proceedings of the American Mathematical Society \\ Providence, Rhode Island, U.S.A. \\ K. FARAHMAND \\ Department of Mathematics \\ University of Ulster \\ Jordanstown \\ CO. Antrim BT $37 O Q B, U K$ \\ E-mail: K.FARAHMAND@ULST.AC.UK \\ FAX NO. +44232 362854
}

Let $(\Omega, A, P)$ be a fixed probability space and let $\left\{g_{j}(\omega)\right\}_{j=1}^{n}$ be a sequence of independent identically distributed random variables defined on $\Omega$. Although there has been considerable attention given to algebraic and trigonometric polynomials with coefficients $g_{j}$ 's, very little is known about the behavior of the random hyperbolic polynomial,

$$
P(x) \equiv P_{n}(x, \omega)=\sum_{j=1}^{n} g_{j}(\omega) \cosh j x .
$$

Denote by $N_{K}(\alpha, \beta)$ the number of real roots of the equation $P(x)=K$ in the interval $(\alpha, \beta)$ and by $E N_{K}(\alpha, \beta)$ its expected value. For the normally distributed $g_{j}$ 's with mean $\mu=0$ and variance one, it is known that $E N_{0}(-\infty, \infty)$ is asymptotic to $(1 / \pi) \log n$, when $n \rightarrow \infty$. This is half that of the random algebraic polynomial $F(x) \equiv F_{n}(x, \omega)=\sum_{j=1}^{n} g_{j}(\omega) x^{j}$ and much smaller than $E N_{0}(0,2 \pi)$ for the random trigonometric polynomial $T(x) \equiv T_{n}(x, \omega)=$ $\sum_{j=1}^{n} g_{j}(\omega) \cos j x$. In fact, although in the interval $(-1,1)$ the hyperbolic polynomial has asymptotically as many zeros as the algebraic polynomial, outside this interval, unlike the algebraic case, the hyperbolic polynomial does not possess any sizable zeros. This could have been caused by (exponentially) fast increase (decrease) of the terms in the hyperbolic polynomial in $(-\infty, 1) \cup(1, \infty)$ which makes the cancellations in this type of polynomial difficult. It is of special interest to establish that for the random hyperbolic polynomial $P(x)$ which of the known patters, if any, $E N_{K}$, for $K \neq 0$, will follow. One would expect that, because of the similarity of the order of $E N_{0}$, the $K$-level crossings be similar to that of the algebraic case. However, our result unexpectedly shows that the $K$-level crossings of the hyperbolic polynomial are similar to that of the trigonometric one. If one classifies the oscillation of different types of polynomials according to the behavior of their real zeros it seems interesting to note that although random hyperbolic polynomials will fall into the algebraic category their properties of $K$-level crossings follow that of the trigonometric case.

This result also motivates to seek the other distinct behavior that the random hyperbolic polynomial may have. It is known that changing $\mu$ from zero to any non-zero bounded will reduce the expected number of real zeros of the random algebraic polynomial by half while not effecting that of the random trigonometric polynomial. For the random hyperbolic polynomial, however, it reduces to $O(1)$, which is obviously not the behavior of the algebraic nor that of the trigonometric polynomial. 


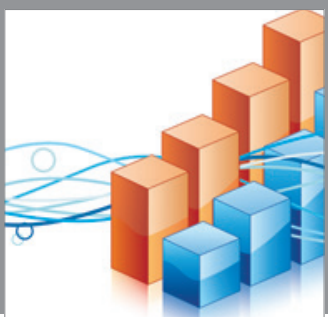

Advances in

Operations Research

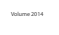

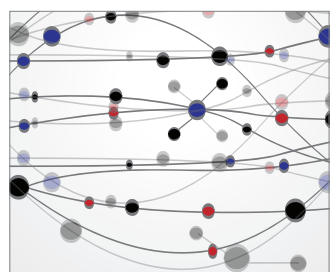

\section{The Scientific} World Journal
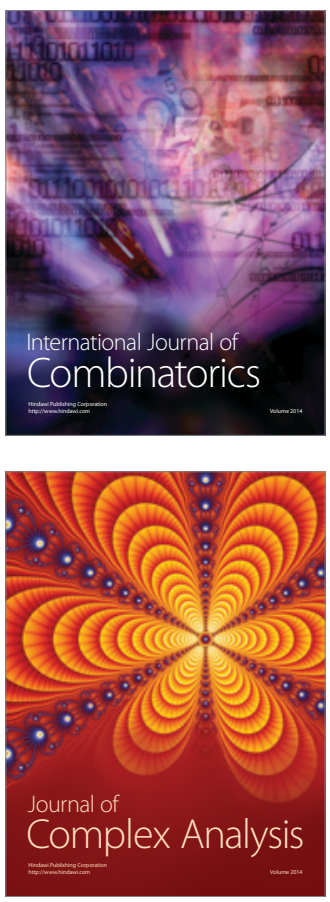

International Journal of

Mathematics and

Mathematical

Sciences
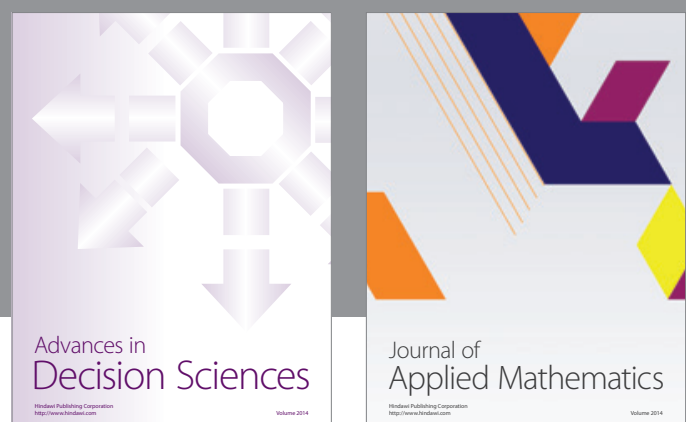

Journal of

Applied Mathematics
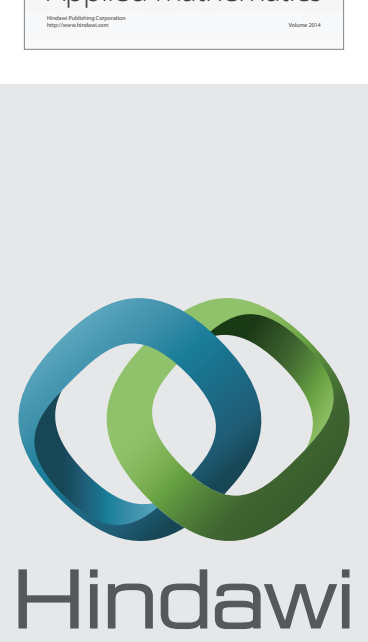

Submit your manuscripts at http://www.hindawi.com
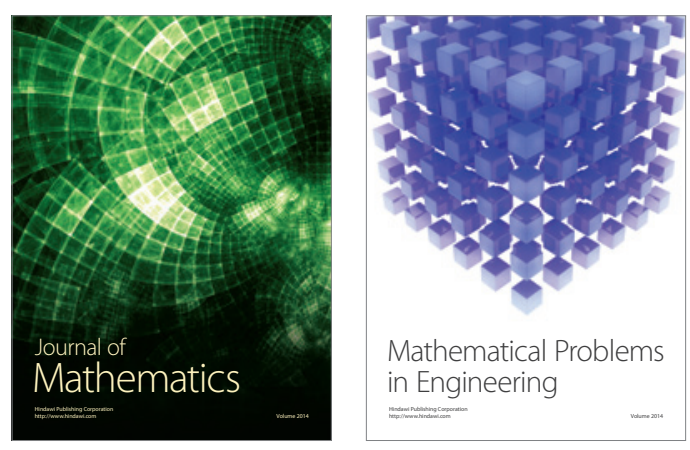

Mathematical Problems in Engineering
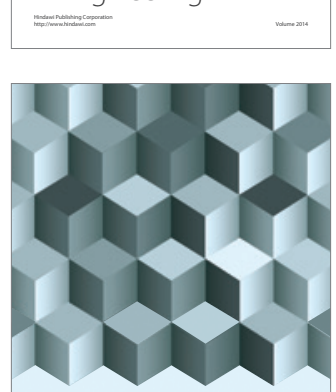

Journal of

Function Spaces
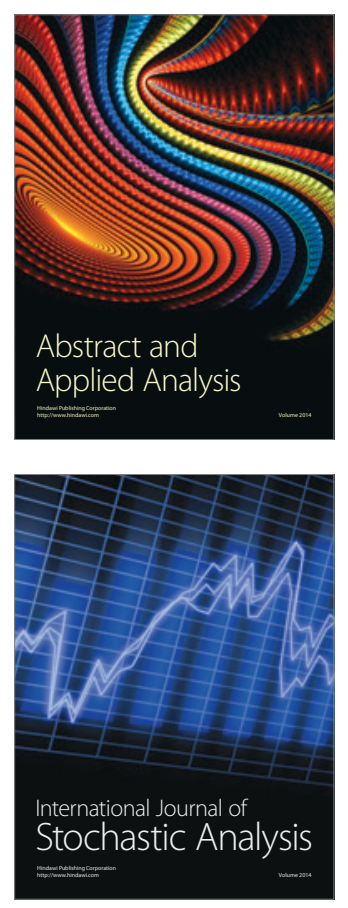

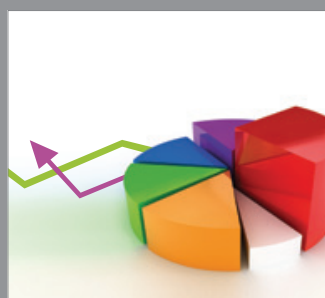

ournal of

Probability and Statistics

Promensencen
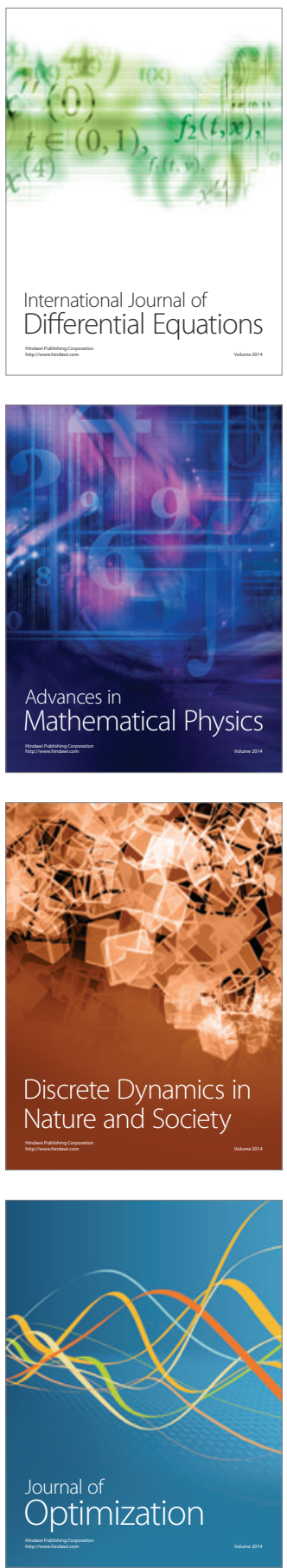